\title{
A Balanced Approach to Traditional Female Genital Surgeries
}

$\mathbf{T}$ raditional genital surgeries are carried out on as many as 114 million women in Africa and parts of the Middle East and Asia. ${ }^{1}$ Traditional cultural justifications of the practice combine religlous and moral beliefs and values. In parts of Africa. variations in the ways the procedures are performed constitute important ethnic differences marking group identities. ${ }^{2}$ In the vast majority of cases. the procedure is carried out on babies or young girls too young to give valid consent. Economic development and increased education of women are influencing current changes in attitudes toward these surgeries but not in simple and direct ways. ${ }^{3}$ The subject is explosive and complex. Traditional female genital surgery is very important in its own right. but it also is a rich illustration of the ethical. political. and medical issues involved in providing. "culturally approprlate care." Too often. culturally appropriate care has been viewed as primarily bearing on patient satisfaction rather than medical outcomes. In the case of these surgeries, it is clear that the issues affect diagnosis. treatment. and management: legal and ethical responsibilities of physicians: and public health policy both locally and worldwide. Western and non-Western health care comprises a set of cultural practices. and culture has direct and practical effects on health.

In this issue, Horowitz and Jackson provide practical information and advice to physicians on traditional female genital surgeries in a way that gets beyond the inflammatory rhetoric that has proven an obstacle to constructive change. ${ }^{4}$ While urging the "need to distinguish respect from unreflective acceptance." the authors explicitly "agree that programs to abolish it should continue." This balance facilitates good medical care for women who have been subjected to these procedures and supports efforts to end these traditional practices. The authors properly anchor their advice in the requirements of good medical practice in the West. A doctor's harsh and insensitive response to a patient's deeply held values generally impedes medical goals. After such a response. patients will refuse to discuss their ideas and intentions openly and fail to return for care. All that is accomplished is a satisfying sense of righteous indignation on the part of the provider.

It is also useful, however, to see these conflicts in their larger historical and cultural context: Western colonialism and the evolution of cultural relativism during the twentieth century. As I sketch this larger context. I will also suggest some ways that I differ in emphasis from Horowitz and Jackson.

Lane and Rubinstein ${ }^{-}$provide a detailed discussion of the historical and philosophical dimensions of this debate. demonstrating that the debate has been polarized along. the lines of ethical universalism versus cultural relativism. Universalist positions posit principles that are true regardless of cultural context. but the principles are usually ones that have developed in a Western intellectual setting. For that reason, these views lend themselves to a form of cultural imperialism echoing colonial expectations that "primitive cultures" must naturally come to accept Western points of view. As a result of the universalizing thetoric of Western reformers. many African and Arab women opposed to these surgeries now see Western reformers as "paternalistic" and "creating a backlash." Universalist opposition to these surgeries has become counterproductive to local efforts to bring them to an end.

Cultural relativism originated in anthropology earlier in this century as a "moral force for tolerance." urging respect for cultural diversity. But over time. especially with the development of postmodernist theory, some approaches to cultural relativism have emphasized the difficulty of true cross-cultural understanding so strongly that they seem to justify any and all local practices as appropriate to the cultures in which they are found.

Middle ground between these extremes is hard to find. espectally in medicine and public health where practical concerns dictate that conclusions will be linked to interventions of some kind. The place to begin. as Horowitz and Jackson demonstrate. is in the language we use to discuss the issues. As Lane and Rubinstein put it. culturally responsive interventions take place "within a complex communicative web." and must acknowledge "that what and how we speak about the practice in question make a real difference. $" \mathrm{~g}$ It is here that I differ with Horowitz and Jackson on emphasis. Granted that African women who endorse the surgeries prefer the English word "circumcision" and are offended by terms such as "genital mutilation." the more neutral term "traditional female genital surgery" seems much more appropriate for medical use. "Circumcision" implicitly seeks to Justify the practice by suggesting a strong analogy to the more widespread practice of male circumcision. That is why it is the preferred term among those who support these traditions. But this analogy is not appropriate except for "mild forms" in which only the clitoral prepuce is cut. Along with their preference for "female circumcision," the authors emphasize that the prevalence of the mild form is unknown and that complications of these mild forms have not been assessed. They quote one patient whose complaint about Western criticism of traditional female genital surgery is based on her own experience of the mild procedure. They also emphasize that only "15 percent of these surgeries include infibulations," that is, the most radical of the procedures. The overall effect of this emphasis is to suggest that these procedures are extremely varied without, at the same time, indicating what forms are actually predominant. Although this emphasis may help to reduce the Westerner's sense of outrage-perhaps a good thing for the clinician - it does not yield an accurate description. Nahid Toubia. the authors source for the 15 percent worldwide prevalence of infibulation, also states 
(on page 10) that mild forms are very uncommon and that 85 percent of traditional female surgeries worldwide involve clitoridectomy. ${ }^{\top}$ This information does not deny that mild forms occur, but any effort to assess the risks of these procedures and the Inagnitude of the problem they constitute requires an accurate appreciation of the great predominance of the more radical forms. The common use of terms such as "savage." "barbaric." "torture." and "child abuse" are obviously inappropriate. They are obstacles to understanding the motivations of parents, the cultures within which they live and raise their families, and the views of individual patients. Achieving balance. however. should not come at the expense of a clear description of the nature of the phenomenon.

Horowitz and Jackson note that "London physicians performed clitoridectomies on refugees in the 1980s"- and Lane and Rubinstein cite the Egyptian Organization on Human Rights to the effect that there have been "big fights among gynecologists, plastic surgeons, and pediatricians [who are] competing to operate and get money from the girls" parents." Both articles also remind the reader that in the 19 th century, and occasionally the first half of the 20 th century. clitoridectomy was used in the United States as medical treatment for a variety of "nervous disorders." At the same time, castration was being used as a treatment for the "disease of masturbation" in males!' - Given that female "circumcision" is now illegal in the United States and Canada, one ought not exaggerate the risk of Western physicians cooperating with parents who request these procedures be done under modern medical conditions. Still. it seems reasonable to urge that. along with sensitivity toward those cultures that see female genital surgeries as necessary to "normality," we should retain the grounds that Western culture provides for holding that such procedures are "wrong."

What grounds are there for taking a strong moral position on these surgeries? And how can such a position avoid unacceptable medical paternalism? Both questions relate to all medical situations in which parents and doctors disagree about what constitutes the best interests of young patients. For example. the Jehovah's Witness parents who refuse medically indicated blood transfusions for their child clash with doctors (and the courts) in a way directly analogous to the parent who seeks medical cooperation in traditional genital surgery for their daughter.

When. if ever. is it right for a "cultural outsider" to impose on a family or society on behalf of individual members of that family or society? The relevant arguments hinge on the idea that actions that involve health risks and/or irrevocably change peoples' bodies (regardless of whether one defines these as "medical") should not be done without informed consent. In the case of traditional genital surgeries. several issues complicate informed consent: the subjects are too young to give consent: the parents who substitute their judgment for their daughters judgment are often not fully informed of consequences. risks, and alternatives; and social pressures around the decision to do the surgeries is so powerful as to call into question the possibility that such consent is freely given. This situation. in turn, raises the question of whether informed consent. always a difficult standard to achieve. is somehow a "Western value" that should not be imposed on others. Lane and Rubinstein cite human rights scholar Alison Slack on this question. identifying "two major opposing concerns; the absolute right of "cultural selfdetermination' and the right of the individual not to be subjected to a tradition or practice that might be harmful or fatal." Behind Slack's statement is a crucial point about human diversity: no matter how homogenous a cultural group may be, there are always individual differences. Regardless of community values. some girls and women - in fact. a substantial number today -in societies that practice these surgeries do not want them. Applying Slack's argument to female genital surgeries is complicated by the fact that, as Horowitz and Jackson make clear, many women explicitly advocate and carry out these surgeries, and many who have been subjected to the procedures express no regret that it has been done.

Of course, it is dangerous to argue that people should be "saved from themselves." that these women do not actually know what their own best interests are. But is that what we do when we argue against these surgeries on a human rights basis? The girls who have the surgeries are too young to consent and are not asked to consent in any event. Further. it is clear that many of those to whom the surgeries appear entirely "normal" are not aware of the numerous medical complications caused by them. Also, false beliefs about female genitalia (such as that an uncircumcised clitoris will "grow long like a penis"] add to the motivation to see the surgeries as both normal and necessary. (Granted that the very possibility of calling a local belief "false" may seem untenable on relativist grounds. but medicine seems to both require and support the idea that at least some beliefs can be shown to be true and others false!) For those adults who had the surgeries as children to say that they are "glad" that they did is not at all the same thing as showing that either as children or as young adults they would have consented to the procedures if fully and accurately informed about them. It seems clear that even parental endorsement and consent to these surgeries is not well informed. nor is it entirely free, given the intense societal pressures. Given the existence of very substantial local resistance to traditional female genital surgeries among women. it seems reasonable to say that the consent issue is one that will stand up to cross-cultural inspection. As to conflict between the "Western" value of autonomy that Horowitz and Jackson contrast to family and community orlented values in other cultures. we should remember that patients can always delegate their autonomy. That is. respect for autonomy should not require patients to make their own decisions - it only insists on allowing them to.

Horowitz and Jackson's study is loaded with good clinical advice that can easily be applied to a varlety of 
cross-cultural situations in clinic. They are grappling with basic issues of ethics and cultural sensitivity in a way that is eminently practical. Understanding that effort. and the reason that so much sensitivity is required. can go far in helping physicians to understand a host of diversity and medical care issues. This is nowhere more important than in those situations where cultural values. gender. and age are simultaneously involved.-Davi J. HuFford,

PHD, Milton S. Hershey Medical Center, Hershey. Pa.

\section{REFERENCES}

1. Kouba L, Muasher J. Female circumcision in Africa: an overview. African Studies Review. 1985:28:95-110.
2. Gruenbaum E. The Islamic movement, development and health education: recent changes in the health of rural women in Central Sudan. Soc Sci Med. 1991:33:637-45.

3. Lane S. Rubinstein R. Judging the other: responding to traditional female genital surgeries. Hastings Center Report. 1996:May-June: $31-40$.

4. Horowitz CR Jackson JC. Female 'circumcision': African women confront American medicine. J Gen Intern Med. 1997:12:491-99.

5. Morsey $\mathrm{S}$. Safeguarding women's bodies: the white man's burden medicalized. Med Anthropol Q. 1991:5:19-23.

6. Toubia N. Women and health in Sudan. In: Toubia N, editor Women of the Arab World: The Coming Challenge. London. En gland: Zed Books: 1988.

7. Toubia N. Female Genital Mutilation: A Call for Global Action. New York. NY: Women Ink: 1993.

8. Engelhardt $H$. The disease of masturbation: values and the concept of disease. Bull Hist Med. 1974:48:231-48. 